$$
\text { UCRL-ID }-119723
$$

\title{
An Overview of EXTOOL: An Analysis Tool for V-TOUGH and NUFT
}

Stephanie Daveler

Lawrence Livermore National Laboratory

Livermore, CA 94551 


\section{DISCLAMMER}

Portions of this document may be illegible in electronic image products. Images are produced from the best available original document. 
, 


\section{Contents}

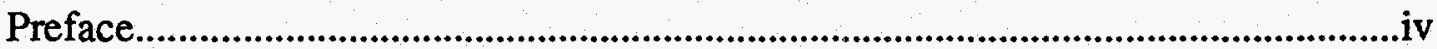

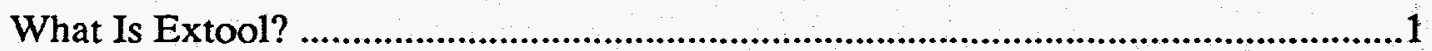

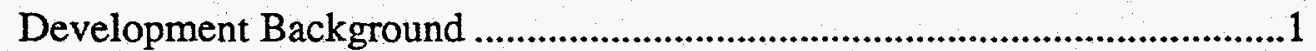

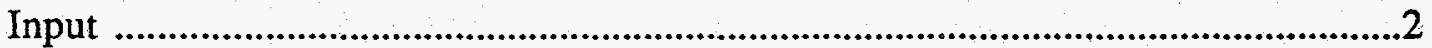

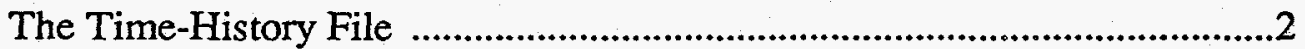

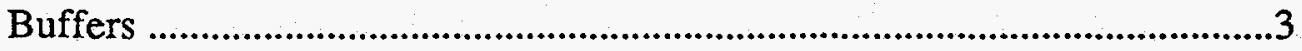

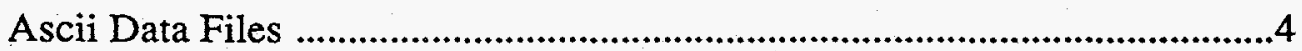

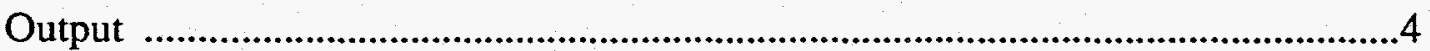

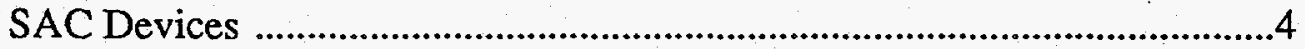

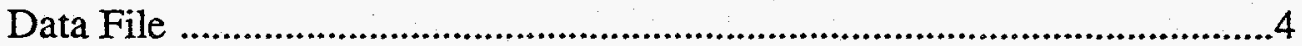

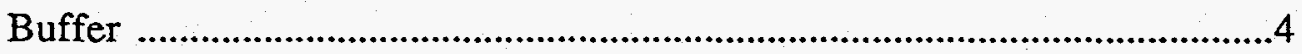

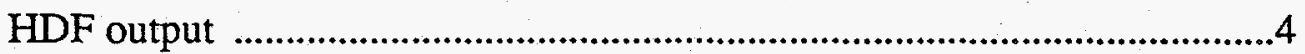

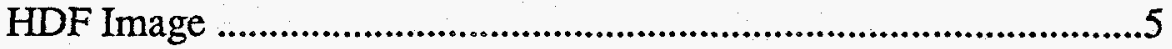

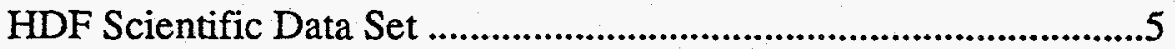

Commands

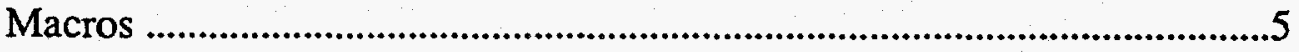

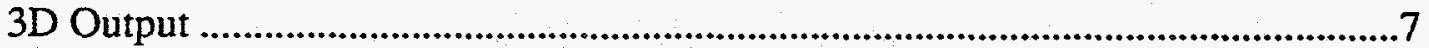




\section{Preface}

This document provides a brief overview of the program Extool. More detailed information can be found in the Extool User's Manual and the Extool Programmer's Guide. The purpose of this document is to give a broad overview of Extool's capabilities and to suggest reasons for using this code instead of another postprocessor. 


\section{Introduction}

Several post-processors have been used in connection with V-TOUGH ${ }^{1}$. Initially, a sequence of utilities were used to extract and plot V-TOUGH information. This changed in 1991 as a new postprocessor, EXTOOL, was developed. Currently, EXTOOL, is the main postprocessor for the modeling codes V-TOUGH and NUFT ${ }^{2}$. In the following sections, a history of V-TOUGH postprocessing is discussed along with an overview of EXTOOL. This overview describes some of Extool's capabilities and suggests reasons for using this code instead of another postprocessor. More detailed information on EXTOOL can be found in the Extool User's Manual and the Extool Programmer's Guide. Both these manuals are drafts, and can be requested by sending email to daveler2@llnl.gov.

\section{What Is Extool?}

Extool, which is short for Extractor Tool, is an application that gathers, manipulates, and displays data. It is written in $\mathrm{C}$ and Fortran. Extool has a graphical user interface, which is implemented using Xview, and must be run in a Window environment. Currently, it has been compiled and run on Sun workstations running OpenWindows.

Extool was designed to quickly obtain, manipulate, and output data from the modeling code V-TOUGH. It is written specifically for V-TOUGH and not as a general postprocessing code. This makes Extool less flexible than other postprocessors but allows for assumptions that increase the speed and ease of use. Some of Extool's capabilities include extracting data from a special output file written by V-TOUGH or NUFT, performing basic data manipulations, and graphically displaying data. Graphics output can also be generated in PostScript format or as a hierarchical data format (HDF) 8-bit image. Data values can be written to disk in an ascii data file or an HDF scientific data set. Information in HDF format can be transferred to more sophisticated data processors. For repetitive operations, there is a simple macro language that can set window items, press buttons, and issue system commands.

\section{Development Background}

Historically, data from V-TOUGH was postprocessed using a number of programs. Because V-TOUGH creates very large ascii data files, the first program used was CNVBIN. CNVBIN was written to find all data in V-TOUGH's output file and store it in an unformatted file. Next, EXTBIN was written to find specific data in the unformatted file and store it in an ascii file. Two programs were written to read the ascii file and produce a graphics file with either a history plot or a contour plot. Another code prints the graphics file, and another displays it. Figure 1 shows the initial postprocessing setup.

1. Modeling code. Vectorized TOUGH (transport of unsaturated groundwater and heat).

2. Modeling code. (Nonisothermal Unsaturated-Saturated Elow and Transport Model). 


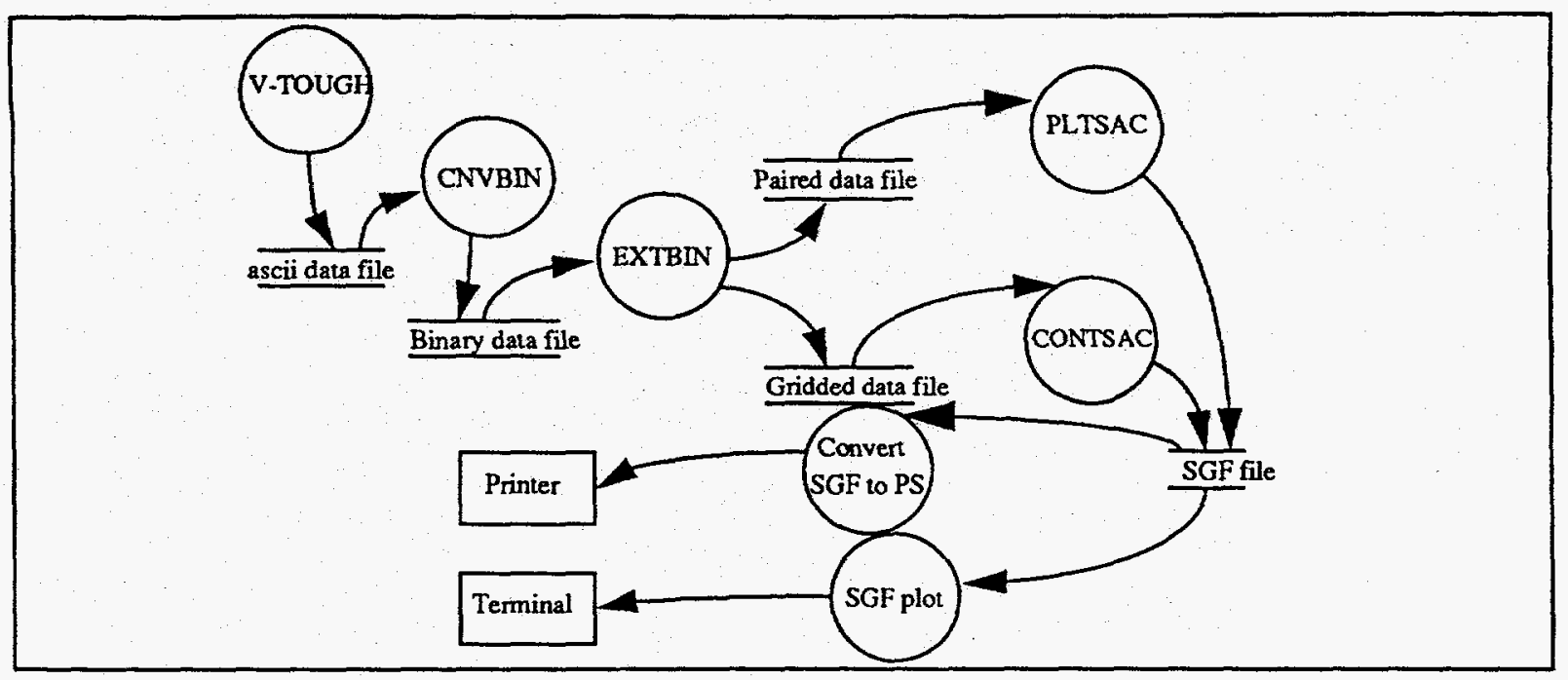

Figure 1. Postprocessing sequence prior to Extool.

One problem with this sequence is the number of transitions needed to convert V-TOUGH's output into a format acceptable to other postprocessors. Another problem is flexibility. The user gets history plots and contour plots. This process can also be slow. A commercial plotting package takes care of some of these problems, but not all of them. We decided to change V-TOUGH's output and to manage the postprocessing using Extool. Data can now flow from the modeling codes V-TOUGH or NUFT as shown in Figure 2. Extool becomes a quick way of viewing data from the modeling codes. More sophisticated data transformations are passed off to commercial products.

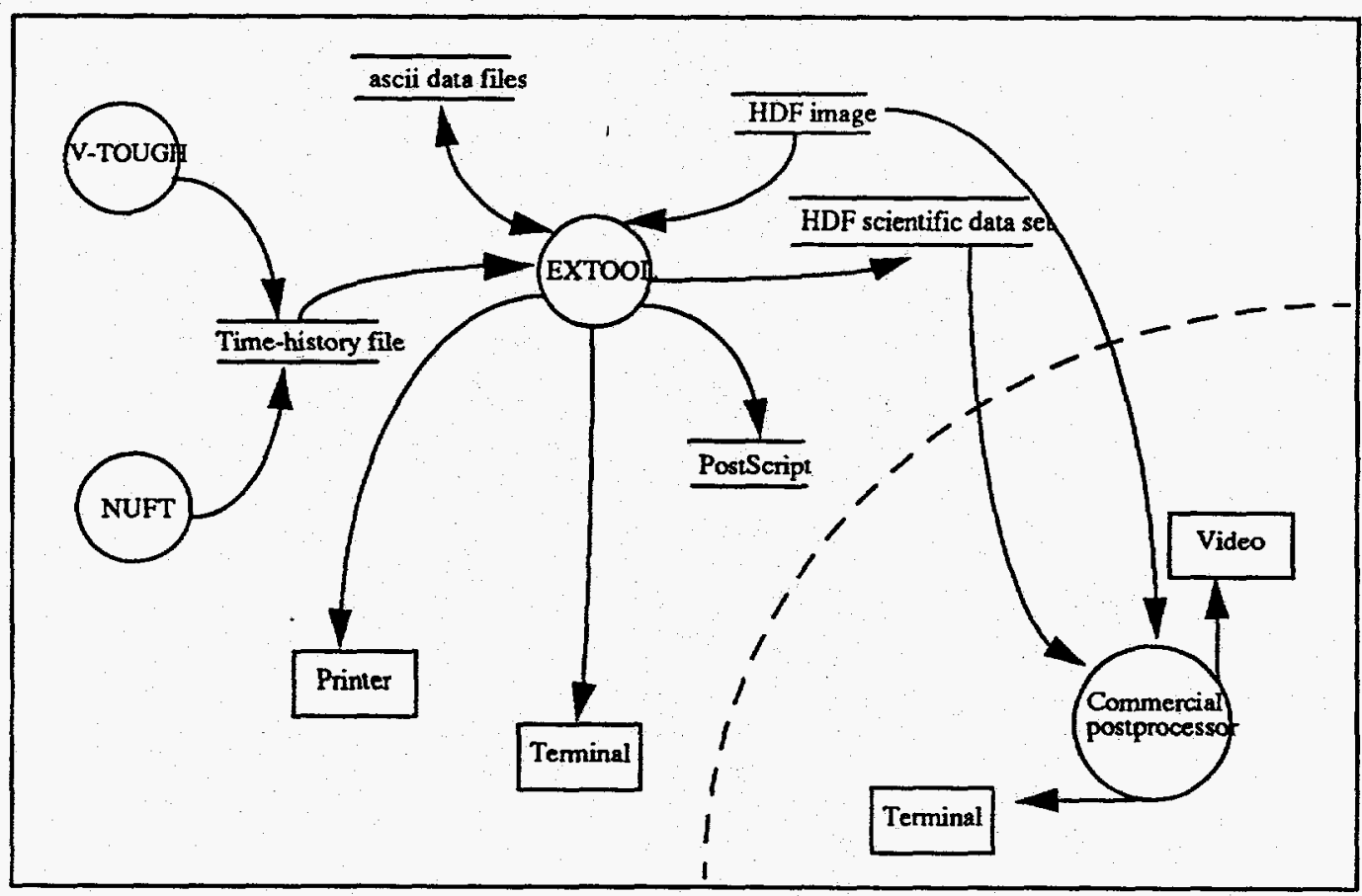

Figure 2. Postprocessing sequence using Extool. 


\section{Input}

Extool was initially designed to extract data from time-history files. To help with data processing, it also accepts several other data formats and can operate on data left in memory. These input options are discussed below.

\section{The Time-History File}

In the context of this document, a time-history file has a special meaning. It is an ascii file created by a modeling code. Both V-TOUGH and NUFT have options to output time-history files. The time-history format was designed to store data using as little space as possible. It consists of header information, followed by data values. Based on the header information, it is possible to identify each data value in the file. The time-history option also gives a user control over which data are saved and when. This is necessary because disk space is limited. Computer models generally have some parameters that change quickly and must be saved frequently to observe their behavior. Other parameters change slowly. To save them frequently wastes disk space.

The time-history option gives a user control over what data to save and when to save them. This is done by specifying extraction sets for a modeling run. An extraction set consists of a set of variables, a set of locations, and a triggering mechanism. Figure 3 shows a sample model domain. At each location, the model calculates parameters such as temperature, pressure, and liquid saturation. It also calculates the velocity in which parameters, such as gas, move from one location to another. These calculations are performed to some specified time limit.

\begin{tabular}{|c|c|c|c|}
\hline Location 1 & Location 5 & Location 9 & Location 13 \\
\hline Location 2 & Location 6 & Location 10 & Location 14 \\
\hline Location 3 & Location 7 & Location 11 & Location 15 \\
\hline Location 4 & Location 8 & Location 12 & Location 16 \\
\hline
\end{tabular}

Figure 3. Model domain.

To capture data for the model domain in Figure 3, a user can define a number of extraction sets. Some examples of extraction sets are shown below. The first example saves everything. This may be too much for a model with a large domain and a long time limit. The second example is more selective.

\section{EXTRACTION SET 1}

Variables: Save all parameters.

Locations: All locations. 
Trigger: At each time step.

EXTRACTION SET 2

Variables: Save temperature.

Locations: At location 11, location 12, location 15, and location 16.

Trigger: When the liquid saturation at location 1 or location 2 exceeds .5 .

\section{Buffers}

When Extool gets data, they are stored internally in a buffer. Normally, this information is removed after Extool completes the specified operation. A user, however, has the option to keep these data in memory and use them as input to another command.

\section{Ascii Data Files}

Extool also accepts ascii data files in PLTSAC and CONTSAC formats, which are the formats used by the postprocessors shown in Figure 1. The PLTSAC format defines paired data sets, and the CONTSAC format defines gridded data sets. Both data types can be specified using a free format, which is flexible and easy to use, and a fixed format, which expects certain information on certain lines. The details of these formats are described in the Extool User's Manual.

An auxiliary program also converts evenly spaced gridded data from an ascii file to a format accepted by Extool. The ascii file must have the format $x$-axis location, $y$-axis location, data value.

\section{Output}

The user has the option to direct Extool command output to different destinations. For history output (paired data), one can select Seismic Analysis $\operatorname{Code}^{1}$ (SAC) devices, a data file, or buffer. For snapshot data (gridded data), the options are SAC devices, data file, buffer, HDF image, HDF scientific data set, or Spyglass ascii. These options are discussed below.

\section{SAC Devices}

The option SAC devices generates graphics output. Graphical output consists of history plots, contour plots, vector plots, and color images. This output can be displayed on a window and written to a disk file. The disk files are unformatted and referred to as SGF files. Extool converts SGF files to PostScript, which is a standard graphics language. An auxiliary program will convert SGF files to encapsulated PostScript. Output from this program has been successfully transferred to FrameMaker, a word processing software.

\section{Data File}

Historically, data were stored in PLTSAC and CONTSAC formats. These formats are still used by our group and are output by Extool. (See the section on Ascii Data Files for more information.)

1. Most of the graphics routines in Extool were created by the developers of LLNL's Seismic Analysis Code. 


\section{Buffer}

(See the section on Buffers for more information.)

\section{HDF output}

The hierarchical data format (HDF) was developed at the National Center for Supercomputing Applications (NCSA) at the University of Illinois at Urbana-Champaign. HDF output is an Extool option and was implemented using a software library developed at NCSA. HDF files are accepted by different software applications and can be converted to other standard formats.

\section{HDF Image}

The HDF image output option generates a color image and is a good otpion for creating movies or videos. This information is written to a file as 8 -bit values. The color table is also stored in the file. $\mathrm{HDF}$ image output can be created for all time steps in the model. The files can then be played back, using the auxiliary program HDFVIEW. This allows a user to view a specific parameter through time or to visually travel through a three-dimensional (3D) grid. Output from this option has been successfully used to generate a video.'

Another auxiliary program, Make_Movie, allows multiple HDF image files, created by Extool, to be displayed on a single canvas. Using this program, a user can show multiple parameters on the same canvas. Optionally, annotations can be added and a new series of HDF image files created.

\section{HDF Scientific Data Set}

The HDF scientific data set is an ascii file. It contains label information, grid dimensions, scales for both axes, and a data value for each location in the grid. This output has been successfully transferred to the Macintosh application Spyglass.

\section{Spyglass Ascii}

Another output option for gridded data is Spyglass ascii. This ascii file contains the location of each grid block and the associated data value. Each line of information describes one grid block. It contains the following values: row distance, column distance, depth, and data value. This data type has not been successfully transferred to another application.

\section{Commands}

Extool performs basic commands on data. It can graphically display history plots, contour plots, grid plots, vector plots, and color images. There are many options that can be set to affect the output of these commands. For more complicated data manipulations, several macro commands are available, as listed below. A special language can also be used to write a script for performing repetitive data processing.

\section{Macros}

All of the available macros are listed below followed by a brief description. A more detailed description can be found in the Extool User's Guide.

1. LLNL Open Computing Center Visualization Laboratory. 
Crossplot

Delta Time

Dimensionless

Liquid Saturation

Interp Time

Line Extremes

Line Tracking

Location Average

Macro Processing

Math

Merge

Null Grid

Plane Extremes
Creates a scatter plot of two paired data sets based on common first-column data values. If first-column data values do not match, command values are calculated using linear interpolation.

Searches a series of snapshot buffers looking for the amount of time each location (grid block) stays within a specified range.

Uses two snapshot buffers. The first must be the initial liquid saturation of a run. The second is a buffer from a later time. The calculation is defined as: $x=\left(\right.$ liquid saturation at time $\left.e_{n}\right)-\left(\right.$ liquid saturation at time $\left.e_{\text {init }}\right)$ if $(x>0.0)$ results $=x /(1-$ liquid saturation at time init $)$

else if $(x<0.0)$ results $=x /($ liquid saturation at time init $)$

else

$$
\text { results }=0.0
$$

Searches through the second column of a paired data set for some occurrence of a value. The corresponding first column value is returned. Linear interpolation is used when necessary.

Finds the value that is the minimum, maximum, absolute minimum, or absolute maximum in a history buffer (paired data).

Calculates the average variable value for a series of lines segments, as they move through time, assuming that water or air is moving through the matrix.

Returns a weighted average using data from a set of grid blocks specified by a user.

Accepts a file with system commands and commands to set and activate window items. Allows for automation of repetitive data manipulations.

Subtracts, multiplies, divides, or adds two data buffers.

Concatenates paired data sets.

Defines null grid blocks. These blocks are not considered when drawing contour, vector, or image plots. Null areas are left blank and outlined with a dashed line.

Finds the value that is the minimum, maximum, absolute minimum, or absolute maximum in a snapshot buffer (gridded data). 
Scale

Slice

Sumflux

Subtracts an offset from input data, then multiplies the resulting values by a scaling factor.

Defines a plane of gridded data, then draws a line through the plane. The values along the line are output as paired data (value, distance). The lines and planes must be parallel to some axis.

Defines a plane of gridded data, then draws a line through the plane. The command sums the perpendicular vectors along the line.

3D HDF Output Calculates an evenly spaced 3D grid.

\section{D Output}

Extool does not project or transform 3D data. Three-dimensional grids are accepted by Extool, but the user must define a plane of interest for most Extool commands. The exception to this is the macro Three-Dimensional HDF Output. This macro creates an evenly spaced 3D grid by interpolating data values. The information is output as an $\mathrm{HDF}$ image data or an HDF scientific data set. A scientific data set is the best way to transfer this information to a product that is meant to operate on 3D data. 


\section{DISCLAIMER}

This report was prepared as an account of work sponsored by an agency of the United States Government. Neither the United States Government nor any agency thereof, nor any of their employees, makes any warranty, express or implied, or assumes any legal liability or responsibility for the accuracy, completeness, or usefulness of any information, apparatus, product, or process disclosed, or represents that its use would not infringe privately owned rights. Reference herein to any specific commercial product, process, or service by trade name, trademark, manufacturer, or otherwise does not necessarily constitute or imply its endorsement, recommendation, or favoring by the United States Government or any agency thereof. The views and opinions of authors expressed herein do not necessarily state or reflect those of the United States Government or any agency thereof. 